\title{
Untersuchungen an Eisbohrkernen von Alpengletschern
}

\section{Einleitung}

Jeder Schneefall enthält Informationen über die Umwelt zur Zeit des Niederschlags. Lösliche Verunreinigungen und Staubgehalt hängen wesentlich von Aerosolen und Staub in der Atmosphäre, und das Verhältnis der stabilen Isotope ${ }^{2} \mathrm{H} /{ }^{1} \mathrm{H}$ und ${ }^{18} \mathrm{O} /{ }^{16} \mathrm{O}$ unter anderem von der Kondensationstemperatur $a b$, um nur zwei Beispiele zu nennen. Im Zuwachsrespektive Akkumulationsgebiet eines Gletschers werden Schneefälle von nachfolgenden Schneefällen überdeckt. Unter dem Druck der darüberliegenden Schichten wird der Schnee zu Firn und Eis verdichtet, und das Eis fließt dann immer tiefer in den Gletscher hinein und zur Abtrags- respektive Ablationszone hin, wo es abschmilzt. Ein Gletscher ist ein Archiv von Schneefällen vergangener Zeiten und damit ein Archiv von Klimadaten.

Die Archivierungszeit ist von Gletscher zu Gletscher verschieden. Typische Zeiten zwischen Schneedeposition in der Akkumulationszone bis zum Abschmelzen in der Ablationszone sind für Alpengletscher Jahrzehnte bis Jahrhunderte. In den großen polaren Eisschilden betragen diese Verweilzeiten bis zu mehreren hunderttausend Jahren. Es ist durchaus möglich, daß auch im Alpengebiet Eisreste aus der letzten Eiszeit überlebt haben, aber Eis, das vor mehr als tausend Jahren gebildet wurde, ist in den Alpengletschern eine große Ausnahme.

Die Qualität der Archivierung ist ebenfalls von Gletscher zu Gletscher verschieden. Wir können die Gletscher für eine Beurteilung grob in zwei Kategorien unterteilen: in temperierte und in kalte Gletscher. In den Alpen dominieren die temperierten Gletscher. Viele Gletscher sind in ihrem obersten Teil kalt, im unteren Teil jedoch temperiert (HAEBERLI, 1976). Temperiertes Eis ist ein Gemisch aus Eis und Wasser, das genau die Gefriertemperatur (beim entsprechenden Druck) aufweist. Das Wasser durchströmt den Gletscher in großen Kanälen und Spalten, es befindet sich aber auch in kleinen Einschlüssen und in einem Netz von Kapillaren, die sich entlang aller Kanten bilden, wo sich drei Eis-Einkristalle treffen. Wasser fließt auch durch dieses fein verästelte System und transportiert lösliche Verunreinigungen und Gase im Gletschereis. Kaltes Eis hat eine Temperatur, die unterhalb des Gefrierpunkts liegt und hat somit keine
Wassereinschlüsse. Kaltes Eis ist impermeabel für Wasser und Luft. Es ist ein wesentlich besseres Archiv als temperiertes Eis.

Sehr kaltes Eis hat den weiteren Vorteil, daß es atmosphärische Luftproben aus der Zeit der Eisbildung archiviert. Falls Schnee ohne Schmelzwasser, also trocken, zu Firn und Eis sintert, wird beim Übergang von Firn zu Eis atmosphärische Luft in Blasen eingeschlossen (STAUFFER, 1985). Falls aber Schnee schmilzt und wieder gefriert, sind die besser löslichen Komponenten der atmosphärischen Luft in den Blasen dieses Eises angereichert (STAUFFER, 1985). Je kälter die mittlere Jahrestemperatur ist, desto geringer ist die Gefahr der Schmelzwasserbildung. Je kälter und je reiner das Eis ist, das eine Blase umschließt, desto geringer ist auch die Gefahr, daß die Zusammensetzung der Luft in den Blasen nach dem Einschluß durch Reaktionen mit Verunreinigungen im Eis verändert wird.

\section{Gewinnung und Untersuchung von Eisbohrkernen}

Altes Eis findet man in der Ablationszone eines Gletschers, am ältesten ist es an der Gletscherzunge. Will man jedoch Eisproben in einer zeitlich kontinuierlichen Stratigraphie, muß man sie durch eine Kernbohrung gewinnen. Mit zunehmender Tiefe stößt die Kernbohrung auf immer älteres Eis. Aus Figur 1 ist ersichtlich, daß bei einer senkrechten Bohrung des Eis je nach Tiefe von verschiedenen Orten des Akkumulationsgebiets stammt, was bei der Interpretation der Resultate zu berücksichtigen ist.

Kernbohrungen in Eis verursachen, bedingt durch die niedrigen Temperaturen und durch das viskose Fließen des Eises, besondere Schwierigkeiten. Es wurden spezielle Bohrtechniken entwickelt. Die Mächtigkeit des Eises von Alpengletschern beträgt höchstens einige hundert Meter. Am Physikalischen Institut der Universität Bern wurde ein Kernbohrer

Bernhard Stauffer, PD, Dr.,

Ulrich Schotterer

Physikalisches Institut der Universität Bern

Sidlerstraße 5, 3012 Bern 


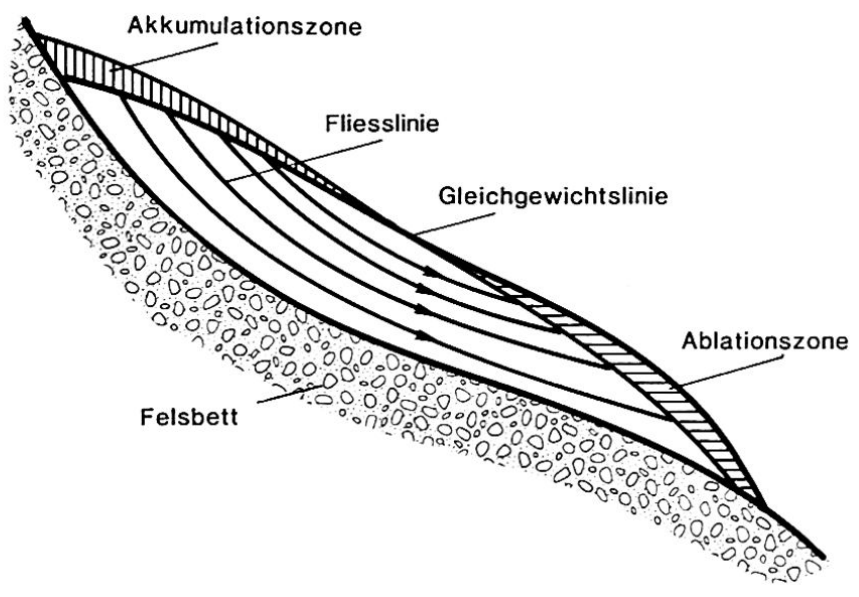

Figur 1 Schematischer Schnitt durch einen Gletscher. Vom höhergelegenen Akkumulationsgebiet fließt das Eis ins tiefergelegene Ablationsgebiet, gelangt dort an die Oberfläche und schmilzt dann $a b$.

konstruiert, der es gestattet, Bohrungen bis rund $100 \mathrm{~m}$ Tiefe durchzuführen (RUFLI, 1976). Das Bohrgerät besteht aus Bohrer, Bohrturm und Kabelwinde und wiegt total rund $200 \mathrm{~kg}$. Es benötigt zusätzlich eine Stromversorgung. Der Bohrer wird an einem Kabel mit elektrischen Leitern ins Bohrloch gesenkt. Der obere Teil des Bohrers besteht aus der Antriebseinheit mit Elektromotor und Getriebe. An der Außenwand sind Kufen angebracht, die beim Starten des Bohrers gegen die Bohrlochwand gepreßt werden, um eine Rotation der Antriebseinheit zu verhindern. Das Kernrohr mit Bohrkrone nimmt Bohrkern und Bohrmehl auf. Pro Bohrvorgang kann ein Bohrkern von rund $1 \mathrm{~m}$ Länge gewonnen werden. Nach jedem Bohrvorgang wird der Bohrer an die Oberfläche gezogen. Das Kernrohr wird vom Bohrer getrennt, und Bohrkern wie Bohrmehl werden entnommen. Die Bohrkerne haben einen Durchmesser von $7,5 \mathrm{~cm}$. Die eigentliche Bohrzeit für eine 100-m-Kernbohrung beträgt einige Stunden. Mit den nötigen Vorbereitungen dauert aber eine Bohrung mehrere Wochen. Erste Messungen an den Eisbohrkernen erfolgen bereits im Feld. Der größte Teil der Eiskerne muß aber in gefrorenem Zustand in die verschiedenen Labors transportiert werden. Dort werden dann analytische Untersuchungen mit modernsten Meßtechniken gemacht. Durchgeführt werden insbesondere:

- Messung der elektrischen Leitfähigkeit am festen Bohrkern (im Feld) als Maß für den Säuregehalt.

- Messung der elektrischen Leitfähigkeit $(\mathrm{Maß}$ für gelöste Verunreinigungen) und des $\mathrm{pH}$-Werts an der geschmolzenen Probe.

- Messung des totalen Staubgehalts und Analyse der Zusammensetzung des Staubs durch Filtration und anschließende Untersuchungen vor allem mit den Methoden der Röntgenfluoreszenz, der Neutronenaktivierung und der Atomabsorption.
- Analyse der löslichen Verunreinigungen durch Ionenchromatographie.

- Messung des totalen Gasgehalts und Analyse der aus Eisproben extrahierten Luft mit Gaschromatographen und Laser-Absorptionsspektrometern.

- Messung von Radioisotopen mit verschiedenen Methoden, unter anderem mit Beschleuniger-Massenspektrometern.

Die erhaltenen Meßergebnisse müssen dann verglichen und entschlüsselt werden, um Klimadaten zu erhalten. Einen kurzen Überblick über den Zusammenhang zwischen Meßgrößen und Klimaparametern findet man bei STAUFFER (1985).

Die Abteilung von Prof. H. Oeschger am Physikalischen Institut der Universität Bern hat in den vergangenen Jahren mehrere Kernbohrungen in temperierten und kalten Gletschern durchgeführt. Die Untersuchungen von Bohrkernen temperierter Gletscher dienen vor allem Untersuchungen spezieller Transportphänomene im Gletscher. Durch Messungen des Gasgehalts und der Gaszusammensetzung kann man beispielsweise abschätzen, wieviel Wasser durch das feine Kapillarsystem im Gletscher fließt (BERNER, 1977). Für die Untersuchungen klimatischer Parameter ist man, wie bereits erwähnt, auf kalte Gletscher angewiesen. Seit 1976 werden vor allem Bohrkerne vom Colle Gnifetti untersucht.

\section{Bohrkerne vom Colle Gnifetti}

Der Colle Gnifetti ist ein Firnsattel auf $4450 \mathrm{~m}$ ü. M., eingebettet zwischen Zumsteinspitze und Punta Gnifetti, im Monte-Rosa-Massiv (Fig. 2). Der Firnsattel ist häufig starken Winden ausgesetzt. Die mittlere Jahrestemperatur beträgt rund $-14^{\circ} \mathrm{C}$. Die mittlere jährliche Zuwachsrate beträgt im Sattel rund $30-40 \mathrm{~cm}$ Wasseräquivalent. Die Zuwachsrate ist nur ein Bruchteil dessen, was als Niederschlag auf dem Colle Gnifetti deponiert wird, da ein großer Teil des Schnees weggeblasen wird, bevor er sich verfestigen kann. Die mittlere Zuwachsrate ändert systematisch über relativ kurze Distanzen (alean, 1983; HaEberli, 1983). Die Feldarbeiten auf dem Colle Gnifetti wurden in Zusammenarbeit mit dem Institut für Umweltphysik der Universität Heidelberg und der Abteilung für Glaziologie der ETH Zürich durchgeführt.

Im Sommer 1976 wurde eine erste Kernbohrung bis $39 \mathrm{~m}$ Tiefe durchgeführt (oESCHGER, 1977). Im Sommer 1977 erfolgte eine zweite Bohrung bis $65 \mathrm{~m}$ Tiefe. Radarmessungen, die 1978 und 1980 durchgeführt wurden, ergaben, daß die Mächtigkeit des Eises im Sattel bis $120 \mathrm{~m}$ beträgt. Im Sommer 1982 wurde dann entlang einer Oberflächen-Fließlinie die Eisschicht an zwei Stellen durchbohrt. Das Felsbett wurde in $124 \mathrm{~m}$, respektive in $66 \mathrm{~m}$, erreicht. 


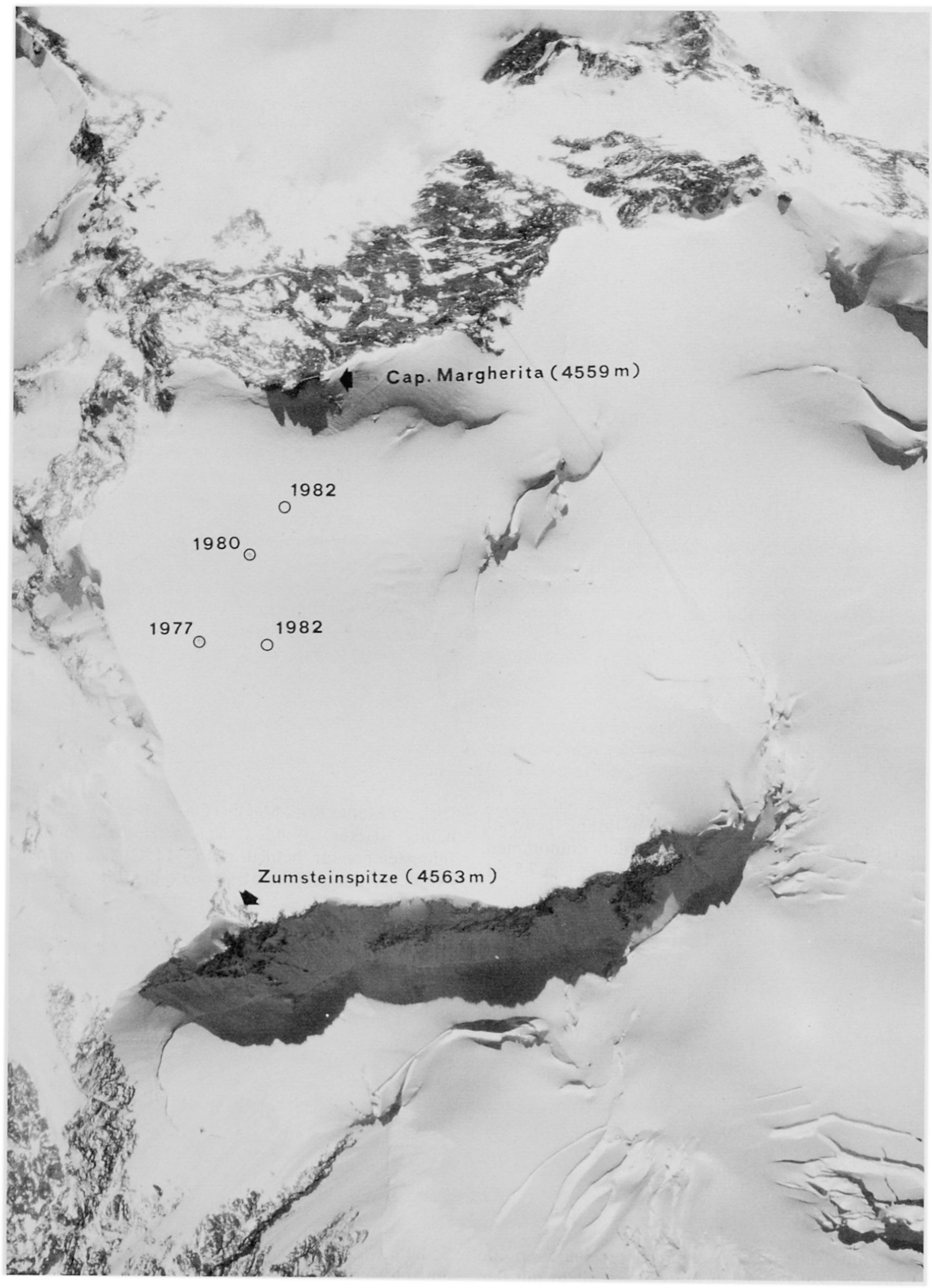

Figur 2 Luftaufnahme von Colle Gnifetti zwischen Capanna Margherita und Zumsteinspitze. Die Jahrzahlen weisen auf die entsprechenden Bohrstellen hin (Luftaufnahme vom 18.8.1980 durch Eidg. Vermessungsdirektion). 
Eine erste Aufgabe besteht in der Datierung der Eiskerne. Es wurden verschiedene Datierungsmethoden verwendet. Bis ins Jahr 1958 zurück ist die Identifikation von Tritiumpeaks, die durch Kernwaffentests verursacht wurden, die sicherste Datierungsmethode (sCHOTTERER, 1981). Markante Staubschichten im Eis können im oberen Teil des Eiskerns historisch erwähnten Sahara-Staubfällen zugeordnet werden und geben für die Datierung zuverlässige Zeitmarken (sCHOTTERER, 1981). Die Messung der ${ }^{210} \mathrm{~Pb}$-Aktivität von Eisproben verschiedener Tiefe erlaubt eine Datierung bis rund 120 Jahre (entsprechen rund $40 \mathrm{~m}$ Tiefe) (GÄGGELER, 1983). Unterhalb $45 \mathrm{~m}$ ist eine Datierung bis heute nur durch eine Schätzung möglich. Die Schätzungen beruhen auf Berechnungen über das Fließverhalten des Eises und gehen von der Voraussetzung aus, daß die Zuwachsraten konstant waren. Aufgrund dieser Schätzungen ist es möglich, daß das Eis des ersten Bohrkerns in $110 \mathrm{~m}$ Tiefe mehr als tausend Jahre alt ist. Die Dicke einer Jahresschicht wäre wegen dem Fließen des Eises auf rund $1 \mathrm{~cm}$ ausgedünnt. Es ist geplant, die tiefsten Schichten mit der ${ }^{14} \mathrm{C}$-Methode zu datieren.

Meerwasser enthält neben den Molekülen, die aus den häufigsten Isotopen bestehen $\left({ }^{1} \mathrm{H}_{2}{ }^{16} \mathrm{O}\right)$, noch $0,015 \%$ Moleküle, die ein schweres Wasserstoffatom $\left({ }^{1} \mathrm{H}^{2} \mathrm{H}^{16} \mathrm{O}\right)$ und $0,204 \%$, die ein schweres Sauerstoffisotop $\left({ }^{1} \mathrm{H}_{2}{ }^{18} \mathrm{O}\right)$ enthalten. Da der Wasserdampfdruck für diese schweren Komponenten des Wassers etwas geringer ist, sind sie im Wasserdampf der Atmosphäre angereichert. Bei der Kondensation werden die schweren Komponenten bevorzugt ausgeregnet. Niederschläge haben bei gegebener isotopischer Zusammensetzung des Wasserdampfes weniger schwere Isotope, je kälter die Kondensationstemperatur ist. Aus diesem Grund sind Winterniederschläge in der Regel isotopisch leichter als Sommerniederschläge. Mit Isotopenuntersuchungen können deshalb Jahresschichten bestimmt werden. Voraussetzung ist, $\mathrm{da} ß$ die Niederschläge saisonal verteilt fallen und in der ursprünglichen Schichtung liegenbleiben. Dies ist auf dem Colle Gnifetti nicht der Fall. Ganze Jahresschichten können aufgewirbelt, durchmischt und wieder deponiert oder weggeblasen werden. Deshalb ist die saisonale Isotopenstratigraphie auf dem Colle Gnifetti nicht vollständig erhalten (sCHOTTERER, 1981).

Die $\delta^{18}$ O-Werte des Eiskerns von 1976 wurden detailliert gemessen. Sie werden benutzt, um Korrelationen mit Temperaturmessungen benachbarter Wetterstationen zu berechnen. Isotopenwerte über mehrere Jahresschichten gemittelt sind in Polargebieten gut korreliert mit lokalen mittleren Jahrestemperaturen (DANSGAARD, 1973). Neuste Untersuchungen zeigen, dass eine solch gute Korrelation während den letzten 70 Jahren auch für den Colle Gnifetti vorhanden ist. Erst die vollständige Analyse des Bohrkerns wird zeigen, ob die gute Korrelation für die ganze
Periode, für die Vergleichsdaten (Instrumentenmessungen, Klimabeobachtungen) vorliegen, gilt. Klimatische Änderungen können bewirken, daß der Anteil von deponiertem zu liegenbleibendem Niederschlag, und damit das Verhältnis Sommer- zu Winterniederschlägen, in der Firnschicht ändert. Dies würde eine Variation der Isotopenwerte bewirken, die nicht direkt durch die mittlere Jahrestemperatur verursacht wird. An den Bohrkernen von 1982 werden die Isotopenverhältnisse gemessen, sobald für den unteren Teil des Bohrkerns eine bessere Datierung vorliegt. Wir erwarten, daß wir die großen Trends der Klimaschwankungen (kleine Eiszeit) in den Isotopendaten finden werden, sind uns aber bewußt, daß eine quantitative Umsetzung der Daten in mittlere Jahrestemperaturen mit großen Unsicherheiten behaftet ist. Falls es sich bei Verdunstung und Kondensation um Gleichgewichtsprozesse handelt, ändern sich $\delta^{2} \mathrm{H}$ und $\delta^{18} \mathrm{O}$ in gleicher Weise. Es gilt:

$\delta^{2} \mathrm{H}=8,0 \times \delta^{18} \mathrm{O}+10 \%$

Wenn man in Niederschlägen größere $\delta^{2} \mathrm{H}$-Werte, bezogen auf die $\delta^{18} \mathrm{O}$-Werte, mißt, spricht man von einem «Deuterium-Exzess» ( ${ }^{2} \mathrm{H}$-Überschuß). Die Ursache für einen solchen Überschuß ist eine sehr schnelle Verdunstung oder eine unvollständige Kondensation, bei denen kinetische Effekte eine Rolle spielen. Bei der Kondensation zu Regen spielen kinetische Effekte keine Rolle, bei der Kondensation zu Schnee können sie eine spielen (JOUZEL, 1982). Für den Colle Gnifetti dürfte der beobachtete ${ }^{2} \mathrm{H}$-Überschuß vor allem dann auftreten, wenn warme und sehr trockene Luftmassen über dem Mittelmeer Wasserdampf aufnehmen. Messungen des ${ }^{2} \mathrm{H}$-Überschusses können dann zur Untersuchung dienen, ob Niederschläge vom Mittelmeer oder vom Atlantik stammen (STICHLER, 1978) (HAEBERLI, 1983).

Die Meßergebnisse der Isotopenuntersuchungen an Bohrkernen vom Colle Gnifetti werden es nicht erlauben, eine detaillierte Geschichte der Jahrestemperaturen zu rekonstruieren. Sie werden aber, in Kombination mit anderen Messungen - insbesondere jenen der Staubkonzentrationen - ermöglichen, auch die Änderungen der Zirkulation und damit die Abfolge von Klimaänderungen zu untersuchen.

Am Colle Gnifetti treten oft Schichten auf, die einen sehr hohen Staubgehalt aufweisen. In den letzten 50 Jahren macht Saharastaub rund zwei Drittel des total deponierten Mineralstaubs aus (WAGENBACH, 1981). In den Schichten mit hohem Staubgehalt, in denen dieser oft über drei Größenordnungen über dem Minimalwert liegt, sind auch die löslichen Verunreinigungen derart hoch, da $\beta$ sie allfällige Signale von Vulkanausbrüchen oder anthropogenen Einflüssen überdecken. Es wurde versucht, den Firnund Eisschichten ganz grob zwei grundsätzlich verschiedene Niederschlagstypen zuzuordnen. 
Sofern der Staubgehalt über $2 \mathrm{mg} / \mathrm{kg}$ Eis beträgt, wird die Schicht dem Typus "Sahara-Staub» zugeordnet. Der deponierte Staub wurde dann (wird angenommen) zusammen mit subtropischen Luftmassen herangeführt, und die in der entsprechenden Firnschicht
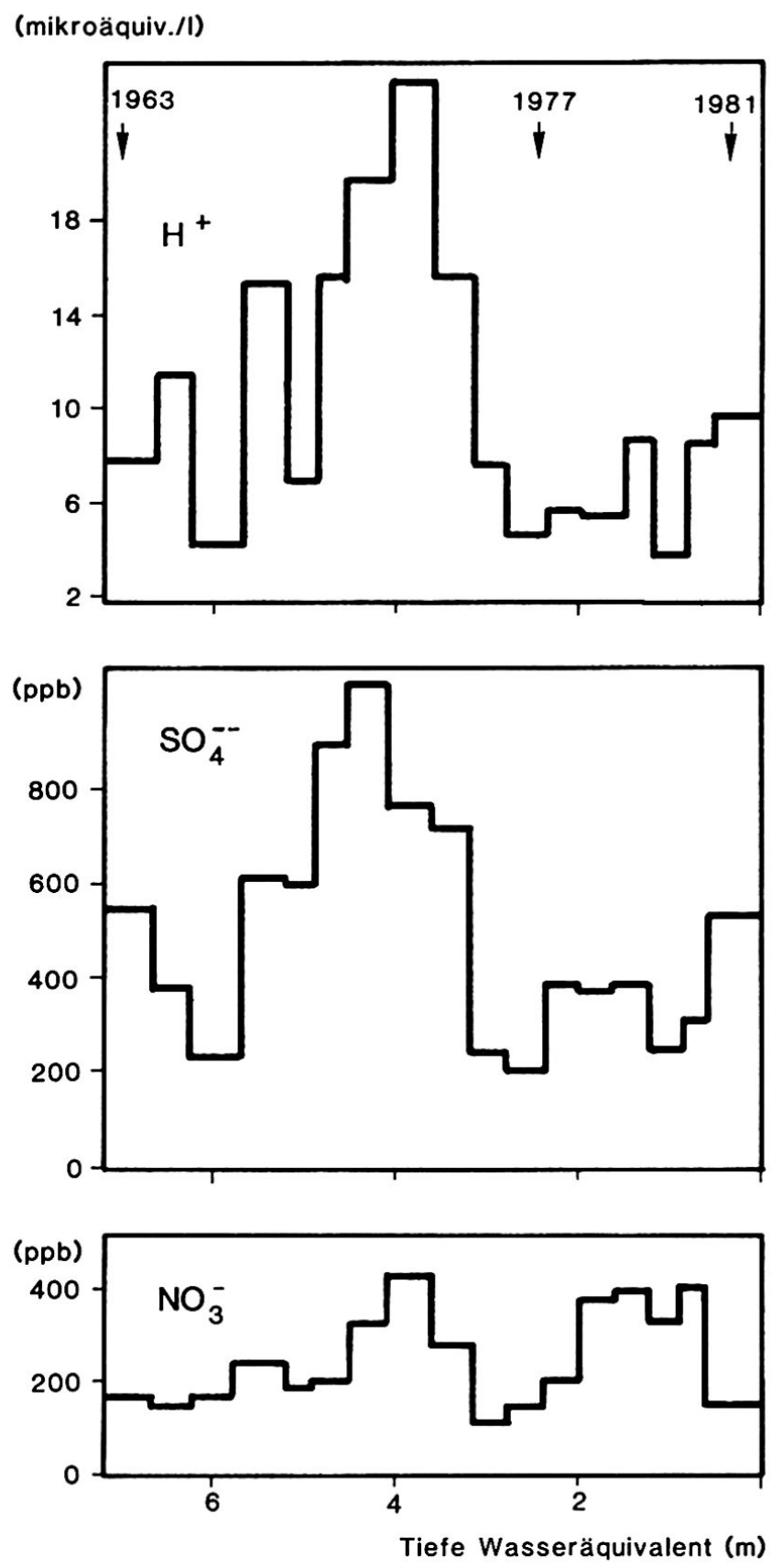

Figur 3 Wasserstoffionen-, Sulfat- und Nitratkonzentration in Firnschichten des Colle Gnifetti, welche Niederschläge der letzten 20 Jahre repräsentieren. Die dargestellten Meßergebnisse sind Mittelwerte aus 8 bis 15 Einzelmessungen. Ergebnisse von staubreichen Schichten wurden nicht berücksichtigt. Nicht alle beobachteten Schwankungen geben Schwankungen der atmosphärischen Konzentration wieder. Wir vermuten aber doch, daß der Rückgang der Sulfationenkonzentration nach 1976 signifikant ist. Er ist möglicherweise auf die internationalen Bemühungen zurückzuführen, den Sulfataussto $B$ zu reduzieren. enthaltenen Verunreinigungen sind nicht repräsentativ für die Verunreinigungen der Troposphäre Mitteleuropas. Im Labor werden Staubschichten über eine TitanKonzentrationsmessung bestimmt (WAGENBACH, 1981). Eine Staubschicht wird als solche definiert, wenn sie mehr als $7 \mu \mathrm{g}$ Titan pro kg Eis enthält. Eine einfache elektrische Leitfähigkeitsmessung, die am gefrorenen Eisbohrkern schon im Feld durchgeführt werden kann, erlaubt es aber, ohne aufwendige Analysen eine grobe Übersicht über die Staubschichten zu erhalten. Fährt man mit zwei Elektroden über die gereinigte Fläche eines Eisbohrkerns, dann ist der gemessene Strom sehr gut mit dem $\mathrm{pH}$-Wert der geschmolzenen Probe korreliert, vorausgesetzt, da $\beta$ der pH-Wert unter 7 liegt (HAMmer, 1980). Bei Vorhandensein von alkalischem Staub wird kein Signal beobachtet (sCHWANDER, 1983). Für den $124 \mathrm{~m}$ langen Bohrkern vom Colle Gnifetti wurden diese Messungen durchgeführt und ausgewertet. Gemäß den Meßergebnissen entfallen rund $15 \%$ des Bohrkerns auf Schichten mit viel Staub, die restlichen $85 \%$ auf Schichten mit lokal repräsentativen Staubkonzentrationen (SCHOTTERER, 1985). An solchen staubarmen Schichten wurden weitere Analysen durchgeführt. Fig. 3 zeigt Meßergebnisse von pH-Messungen (gemessen mit pH-Elektrode an der geschmolzenen Probe) und von $\mathrm{SO}_{4}=$ und $\mathrm{NO}_{3}{ }^{-}$Analysen, gemessen mit einem Ionenchromatographen. Für größere Tiefen liegen erst vereinzelt Meßwerte vor. Ein Mittelwert für 16 Proben aus einer Tiefe, die einer Zeit um 1860 entspricht, ergibt für $\mathrm{SO}_{4}=$ eine Konzentration von rund $90 \mathrm{ppb}\left(\mu \mathrm{g} / \mathrm{kg}\right.$ Eis), für $\mathrm{NO}_{3}{ }^{-}$von rund $65 \mathrm{ppb}$ und für den $\mathrm{pH}$-Wert rund $3 \mu$ äquiv./l. Es muß darauf hingewiesen werden, daß zwar die Verunreinigungen im Eis stark von den Verunreinigungen der Atmosphäre abhängen, daß aber kein einfacher Zusammenhang zwischen den beiden Parametern besteht. Schwankungen der Ionenkonzentrationen im Eis lassen nicht ohne weiteres auf Schwankungen dieser Konzentrationen in der Atmosphäre schließen.

Dennoch sei hier ein Vergleich der Daten von Fig. 3 mit den vorindustriellen Werten erlaubt. Der Anstieg zu den deutlich höheren Werten im oberen Kernabschnitt müssen auf anthropogene Einflüsse zurückgeführt werden. In Fig. 4 sind die Leitfähigkeitsmessungen am gefrorenen Bohrkern für die oberen $45 \mathrm{~m}$ und die pH-Werte der geschmolzenen Proben der obersten $10 \mathrm{~m}$ dargestellt. Der Anstieg des Säuregehalts ist auch hier sehr deutlich sichtbar.

\section{Vergleich mit Ergebnissen polarer Eisbohrkerne}

Im Zentrum der großen polaren Eisschilde entspricht die Zuwachsrate annähernd der Niederschlagsrate. Obwohl auch hier sehr starke Winde auftreten, ist die Ablagerung und Schichtung regelmäßiger. Der Trans- 
portmechanismus für Niederschläge und Schadstoffe ist grundsätzlich verschieden. Bei den Eisschilden handelt es sich um Hochplateaus; lokale vertikale Luftbewegungen spielen eine kleine Rolle. Der Colle Gnifetti ist ein Hochgebirgssattel, der von Luftmassen umströmt oder überströmt werden kann. Lokale vertikale Luftströmungen spielen für den Transport von Wasserdampf und Aerosolen eine bedeutende Rolle (WAGENBACH, 1985).

Die Korrelation der mittleren $\delta^{18} \mathrm{O}$-Werte mit lokalen mittleren Jahrestemperaturen kann noch nicht verglichen werden, da zu wenig Messungen vom Colle Gnifetti vorliegen. Es muß insbesondere noch abgeklärt werden, ob es sinnvoll wäre, auch Isotopenanalysen getrennt für Staubschichten und für staubarme Schichten durchzuführen.

Ein anthropogen bedingter Anstieg von $\mathrm{SO}_{4}=$ und $\mathrm{NO}_{3}{ }^{-}$wurde auch in polaren Eiskernen gemessen. In einem Bohrkern von Südgrönland stieg die Sulfationenkonzentration von 45 ppb um 1900 auf rund 85 ppb Ende der siebziger Jahre, die Nitrationenkonzentration im gleichen Zeitraum von $45 \mathrm{ppb}$ auf rund $80 \mathrm{ppb}$ (NEFTEL, 1985). Die bisher gemessenen vorindustriellen Sulfationen- und Nitrationen-Konzentrationen am Colle Gnifetti scheinen höher als in Südgrönland zu sein, was durch die kürzere Distanz $\mathrm{zu}$ natürlichen Quellen bedingt sein kann. Der Konzentrationsanstieg, relativ zum vorindustriellen Wert, ist für den Colle Gnifetti für Sulfationen und Nitrationen rund doppelt so groß wie für Südgrönlandeis. Dieser stärkere Anstieg überrascht nicht, da der Colle Gnifetti näher bei den anthropogenen Quellen liegt.

Erste Analysen der aus Eisproben vom Colle Gnifetti extrahierten Luft zeigen, daß der $\mathrm{CO}_{2}$-Gehalt gegenüber dem atmosphärischen Wert stark angereichert ist. Eisproben aus polaren Eisschilden zeigen den gleichen Effekt, falls die mittlere Jahrestemperatur gleich hoch ist. Ursache ist Schmelzwasser, das im Hochsommer gebildet wird. Andere Spurengase sind weniger empfindlich auf Schmelzwassereinflüsse. Wir werden beispielsweise prüfen, ob sich Eis vom Colle Gnifetti eignet, die Entwicklung der atmosphärischen Methankonzentration in Mitteleuropa zu rekonstruieren.

Polare Eisproben erlauben es, Klimaparameter über die letzten 200000 Jahre zu untersuchen. In Alpengletschern wird man sich auf die letzten tausend Jahre beschränken müssen. Für die Untersuchung vieler globaler Parameter, wie beispielsweise der $\mathrm{CO}_{2}$-Konzentration der Atmosphäre, ist man auf polare Eisproben angewiesen. Es ist aber zu bedenken, daß viele Klimadaten, die aus Eiskernen gewonnen werden, regionalen Charakter haben (Niederschlagsrate, Temperatur) und daß bei gewissen anderen zumindest eine Breitenabhängigkeit vermutet wird.

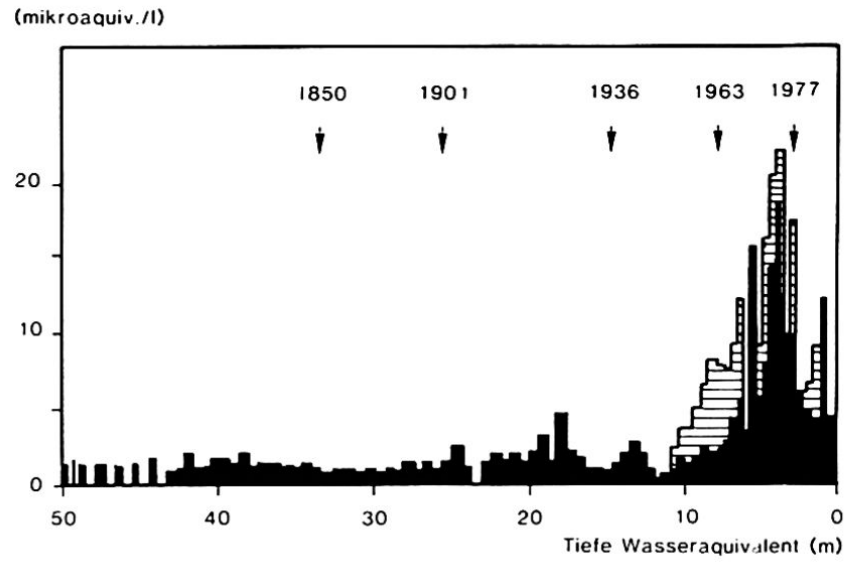

Figur 4 Wasserstoffionenkonzentration, bestimmt durch el. Leitfähigkeitsmessungen am gefrorenen Eis (schwarzes Balkendiagramm). Für die oberen $10 \mathrm{~m}$ liegen auch Meßresultate des $\mathrm{pH}$-Werts, gemessen an den entsprechenden geschmolzenen Proben, vor (schraffiertes Balkendiagramm). Die Jahrzahlen kennzeichnen Datierungsmarken (Tritiummaximum resp. Staubschichten). Von rund 1950 an (entspricht $12 \mathrm{~m}$ Tiefe) beginnen die Werte infolge anthropogener Einflüsse schnell anzusteigen.

Untersuchungen von Eisbohrkernen von Alpengletschern sind nicht nur eine Ergänzung zu den Untersuchungen an polaren Kernen, sondern viele Fragen betreffend Breitenabhängigkeit oder Abhängigkeit von der Distanz zur Quelle können nur durch solche Untersuchungen beantwortet werden. Die hochgelegenen, kalten Gletscher der mittleren Breiten werden in Zukunft als äußerst zuverlässige Archive von Klimadaten eine zunehmende Bedeutung erlangen. Die Umsetzung dieser Archivdaten in beispielsweise mittlere Jahrestemperaturen und Niederschlagsraten ist zur Zeit noch mit gewissen Unsicherheiten verbunden; eine zuverlässige Interpretation wird jedoch angestrebt.

\footnotetext{
Abstract

Cold alpine glaciers are archives of climatic parameters. Core drilling in alpine glaciers allows to collect ice samples of ages up to 1000 years. First results from analyses of an ice core from Colle Gnifetti (Swiss Alps) show already interesting results. Frequent occurring dust bands contain mainly dust from the Sahara and are therefore characteristic for precipitations transported with subtropic air masses. An increase of the acidity of the precipitations starting at the beginning of our century is most probably an anthropogenic effect. For the upper $10 \mathrm{~m}$ of the ice core, $\mathrm{SO}_{4}=$ and $\mathrm{NO}_{3}{ }^{-}$concentrations have also been measured.
} 


\section{Literatur}

ALEAN, J., HAEBERLI, W., SCHÄDLER, B. (1983): Snow Accumulation, Firn Temperature and Solar Radiation in the Area of the Colle Gnifetti Core Drilling Site (Monte Rosa, Swiss Alps): Distribution Patterns and Interrelationships. In: Zeitschrift für Gletscherkunde und Glazialgeologie 19(2), 131-147.

BERNER, W., STAUFFER, B., OESCHGER, H. (1977): Dynamic Glacier Flow Model and the Production of Internal Meltwater. In: Zeitschrift für Gletscherkunde und Glazialgeologie 13, 209-217.

DANSGAARD, W., JOHNSEN, S.J., CLAUSEN, H.B., GUNDESTRUP, N. (1973): Stable Isotope Glaciology, Meddelelser om Grönland 197(2)

GÄGGELER, H., VON GUNTEN, H.R., RÖSSLER, E., OESCHGER, H., SCHOTTERER, U. (1983): ${ }^{210} \mathrm{~Pb}$-Dating of Cold Alpine Firn/lce Cores from Colle Gnifetti, Switzerland. In: Journal of Glaciology 29(101), 165-177.

HAEBERLI, W. (1976): Eistemperaturen in den Alpen. In: Zeitschrift für Gletscherkunde und Glazialgeologie 11(2)

HAEBERLI, W., SCHOTTERER, U., WAGENBACH, D., HAEBERLI-SCHWITTER, H., BORTENSCHLAGER, S. (1983): ACCUmulation Characteristics on a Cold, High-Alpine Firn Saddle from a Snow-Pit Study on Colle Gnifetti, Monte Rosa, Swiss Alps. In: Journal of Glaciology 29 (102), 260-271.

HAMMER, C.U. (1980): Acidity of Polar Ice Cores in Relation to Absolute Dating, Past Volcanism, and Radio-Echoes. In: Journal of Glaciology 25(93), 359-372.

JOUZEL, J., MERLIVAT, L., LORIUS, C. (1982): Deuterium Excess in an East Antarctic Ice Core Suggests Higher Relative Humidity at the Oceanic Surface During the Last Glacial Maximum. In: Nature 299, 688-691.

NEFTEL, A., BEER, J., OESCHGER, H., ZÜRCHER, F., FINKEL, R.C. (1985): Sulphate and Nitrate Concentrations in Snow from South Greenland 1895-1978. In: Nature 314(6012), 611-613.

OESCHGER, H., SCHOTTERER, U., STAUFFER, B., HAEBERLI, W., RÖTHLISBERGER, H. (1977): First Results from Alpine Core Drilling Projects. In: Zeitschrift für Gletscherkunde und Glazialgeologie 13, 193-208.

RUFLI, H., STAUFFER, B., OESCHGER, H. (1976): Lightweight 50-Meter Core Drill for Firn and Ice. In: Ice-Core Drilling, University of Nebraska Press 133-153.
SCHOTTERER, U., HAEBERLI, W., GOOD, W., OESCHGER, H., RÖTHLISBERGER, H. (1981): Datierung von kaltem Firn und Eis in einem Bohrkern vom Colle Gnifetti, Monte Rosa. In: Jahrbuch der Schweizerischen Naturforschenden Gesellschaft, wissenschaftl. Teil 1978, 48-57.

SCHOTTERER, U., OESCHGER, H., WAGENBACH, D., MÜN$\mathrm{NICH}$, K.O. (1985): Information on Paleo-Precipitation on a High-Altitude Glacier. In: Zeitschrift für Gletscherkunde und Glazialgeologie 21.

SCHWANDER, J., NEFTEL, A., OESCHGER, H., STAUFFER, B (1983): Measurements of Direct Current Conductivity on Ice Samples for Climatological Applications. In: Journal of Physical Chemistry 87, 4157-4160.

STAUFFER, B., NEFTEL, A., OESCHGER, H., SCHWANDER, J. (1985): $\mathrm{CO}_{2}$ Concentration in Air Extracted from Greenland Ice Samples. In: Greenland Ice Core: Geophysics, Geochemistry and the Environment, AGU Geophysical Monograph 33.

STAUFFER, B. (1985): Klimainformationen aus Eisbohrkernen. In: Jahrbuch der Schweizerischen Naturforschenden Gesellschaft, wissenschaftlicher Teil 1983, 38-47.

STICHLER, W., HERRMANN, A. (1978): Räumlich-zeitliche Variationen der Isotopengehalte randalpiner Niederschläge. 15 Intern. Tagung für alpine Meteorologie, Grindelwald 1978, 1. Teil Veröff. Schweiz. Meteorol. Zentralanstalt 40, 271-275.

WAGENBACH, D. (1981): Pilotstudie zur Aerosoldeposition auf einer hochalpinen "kalten" Firndecke. Dissertation, Universität Heidelberg.

WAGENBACH, D., GORLACH, U., HAFFA, K. JUNGHANS, H. G., MÜNNICH, K. O., SCHOTTERER, U. (1985): A Longterm Aerosol Deposition Record in a High Altitude Alpine Glacier. WM Technical Conference on Observation and Measurements of Atmospheric Contaminants (TECOMAC) Vienna 1983 (in press).

Kernbohrung und Bohrkernuntersuchungen wurden gemeinsam mit dem Institut für Umweltphysik der Universität Heidelberg durchgeführt. Unsere Mitarbeit wurde durch die finanzielle Unterstützung durch den Schweizerischen Nationalfonds ermöglicht. Wir danken D. Wagenbach für Diskussionen und die Durchsicht des Manuskripts. 